\title{
Predicting Risk Propagation of Corporate Internet Reporting Based on Fuzzy Neural Network
}

\author{
Lingyan $\mathrm{Ou}^{1,2^{*}}$, Ling $\mathrm{Chen}^{3}$ \\ ${ }^{1}$ School of Accounting, Fujian Jiangxia University, Fuzhou 350108, China \\ ${ }^{2}$ Finance \& Accounting Research Centre, Fujian Province Philosophy Social Science Research Base, Fuzhou 350000, China \\ ${ }^{3}$ Risk \& Security Center, Alibaba Local Life Group, Shanghai 200000, China
}

Corresponding Author Email: oly013@fjjxu.edu.cn

https://doi.org/10.18280/isi.250411

Received: 19 April 2020

Accepted: 28 June 2020

\section{Keywords:}

corporate internet reporting $(C I R)$, risk propagation, fuzzy neural network (FNN), evaluation index system (EIS)

\begin{abstract}
With the rapid advances of Internet technology, some listed companies choose to disclose their financial information in the form of corporate internet reporting (CIR). However, there is little report on the risk factors and formation mechanism of CIR risks. To better prewarn, prevent and regulate CIR risks, this paper designs an CIR risk propagation model based on fuzzy neural network (FNN). Firstly, an evaluation index system (EIS) was established for CIR safety, and subject to fuzzy comprehensive evaluation (FCE), after reliability analysis and weighting of the indices. Based on the evaluation results, the hypotheses and risk propagation mode were summarized, and used to set up a risk propagation model. Finally, a neural network (NN) algorithm was created to predict the CIR risk propagation path. The proposed model and algorithm were proved effective through experiments. The research findings provide a novel tool to dig deep into the propagation mechanism of CIR risks.
\end{abstract}

\section{INTRODUCTION}

The rapid advances of Internet technology have greatly changed the financial information disclosure. Some listed companies choose to disclose their financial information in the form of corporate internet reporting (CIR) on various online platforms [1-4]. Compared with traditional paper report, the CIR is open, transparent, and standardized, providing a wide audience with sufficient information in time. Like other online information, the CIR also faces various risks, which are amplified by people's behaviors, values, interpretation, and association [5-9].

There are at least two kinds of CIR risks: the objective loss of participants on the information transmission chain, and the negative impacts of cultural and social factors involved in the disclosure [10-14]. Chen [15] constructed an evaluation index system (EIS) for CIR quality, evaluated the CIR quality of a listed company in northeastern China's Liaoning Province, and put forward some improvement measures, highlighting the ability of extensible Business Reporting Language (XBRL) to automatically exchange and extract financial information in any software and information system, and make financial information more reliable and accurate. Tsantekidis et al. [16] studied the propagation mechanism of systemic financial risks, sorted out the influencing factors of systemic risks, and gave corresponding prevention suggestions.

The CIR in XBRL enables convenient and quick search, extraction, analysis, sharing, and mining of high-quality financial information. However, the ability to prevent and control CIR risks needs to be further enhanced in actual application [17-23]. Cakra and Trisedya [24] compared XBRL with Electronic Data Interchange (EDI) in operation mechanism and technical features, and summarized the positive effects of Internet security techniques, e.g. Internet protocol, encryption technology, digital signature, and virtual private network (VPN), on the implementation of XBRL. Shan [25] identified and classified the risk factors of XBRL CIR of wind power companies, constructed a Net draw-based network model of the risk propagation path, and controlled the CIR risks in the disclosure phase and the decision-making phase.

The existing studies on risk propagation path mainly tackle the maintenance of systematic financial security, and the financial security of largescale engineering projects. Only a few scholars have explored the influencing factors and formation mechanism of CIR risks. Moreover, there is little report that evaluates CIR risks, in virtue of the strengths of neural networks (NNs) in adaptation and self-learning. To improve the effectiveness and accuracy of risk warning, crisis prevention and financial supervision of the CIR, this paper presents a risk propagation model of the CIR based on fuzzy neural network (FNN).

The remainder of this paper is organized as follows: Section 2 constructs an EIS for CIR safety, performs reliability analysis and weighting of the indices, and makes a fuzzy comprehensive evaluation (FCE) of the EIS; Section 3 summarizes the hypotheses and risk propagation mode based on the evaluation results, and derives the risk propagation model in a systematic manner; Section 4 builds up a backpropagation NN (BPNN) algorithm capable of predicting the risk propagation path of the model, and specifies the training process of the network; Section 5 verifies the effectiveness of the proposed model and algorithm; Section 6 puts forward the conclusions of this research. 


\section{CIR SAFETY EVALUATION}

\subsection{EIS construction}

The EIS of CIR safety is the prerequisite for exploring the propagation mechanism of CIR risks. Considering the various objectives of CIR safety evaluation, some CIR safety indices were synthetized as per the design principle of CIR system, and in accordance with the requirements of documents like Electronic Information Disclosure Specification for Listed Companies and Classification of Information Disclosure for Listed Companies. In this way, a four-layer EIS was established for CIR safety, including three primary indices: CIR information security, CIR tool security, and CIR security system. Each primary index has several secondary and tertiary indices. The hierarchical EIS is detailed as follows:

The first layer (goal):

$A=\{$ CIR safety $\}$.

The second layer (primary indices):

$A=\{A 1, A 2, A 3\}=\{\mathrm{CIR}$ information security, CIR tool security, CIR security system $\}$.

The third layer (secondary indices):

$A 1=\{A 11, A 12, A 13, A 14\}=\{$ General information security, Information security of financial statement, Information security of corporate governance and internal control, Information security of auxiliary and forecast $\}$;

$A 2=\{A 21, A 22\}=\{$ Security of financial data disclosure form, Security of CIR tool $\}$;

$A 3=\{A 31\}=\{$ Completeness of security assurance system $\}$.

The fourth layer (tertiary indices)

$A 11=\{A 111, A 112, A 113, A 114\}=\{$ Partner information, Affiliated entities, Information of development strategies, Information of employee contacts $\}$;

$A 12=\{A 121, A 122, A 123, A 124, A 125\}=\{$ Details of annual financial information, Details of semi-annual financial information, Details of quarterly financial information, Details of monthly financial information, Internal financial analysis report $\}$;

$A 13=\{A 131, A 132, A 133, A 133, A 134, A 135, A 136, A 137$, $A 138, A 139\}=\{$ Details of shareholders and equity changes, Information of executives, Work reports of independent directors, Detailed rules of corporate management, Details of integrity record, Public resolutions of shareholder meeting, Internal agenda of shareholder meeting, Self-evaluation report on internal control, Audit report on internal control $\}$;

$A 14=\{A 141, A 142, A 143, A 144, A 145, A 146, A 147$, $A 148\}=\{$ Information of related-party transaction, Information of fundraising projects, Information of non-fundraising projects, Investment information, Information of profit distribution, Information of major events, Information of profit and cost forecast, Information of stock exchange $\}$;

$A 21=\{A 211, A 212, A 213, A 214\}=\{\mathrm{XBRL}$ report, Chart format of financial data, Form of online interactions, Online and offline bulletin boards $\}$;

$A 22=\{A 221, A 222, A 223, A 224, A 225, A 226\}=\{$ Links to stock exchanges, Links to other websites, Content selection menu \& site map, Internal search engine, Download tools, Data processing tools $\}$;
$A 31=\{A 311, \quad A 312, \quad A 313, \quad A 314, \quad A 315\}=\{$ Archival information of Corporate Internet Reporting (CIP), Information of Internet security report, Information of user privacy statement, Information of safety tips and copyright statement, Certification of credible website\}.

\subsection{Reliability analysis and weighting}

Before reliability analysis, the proposed EIS was subject to consistency and stability tests on SPSS 21.0. As shown in Table 1, the Cronbach's alpha of each index was greater than 0.70 , indicating that the EIS has good overall reliability. According to the reliability analysis results of tertiary indices, the tertiary indices under secondary index $A 14$ Information security of auxiliary and forecast were adjusted as Information of related-party transaction, Information of fundraising projects, Information of non-fundraising projects, Investment information, and Information of profit distribution; the tertiary indices under secondary index $A 13$ Information security of corporate governance and internal control was adjusted as Information of executives, Detailed rules of corporate management, Details of integrity record, Internal agenda of shareholder meeting, Self-evaluation report on internal control, and Audit report on internal control.

Table 1. The results of reliability analysis on secondary indices

\begin{tabular}{ccc}
\hline Secondary indices & Cronbach's alpha & Number of terms \\
\hline$A_{11}$ & 0.717 & 58 \\
$A_{12}$ & 0.792 & 10 \\
$A_{13}$ & 0.821 & 44 \\
$A_{14}$ & 0.834 & 8 \\
$A_{11}$ & 0.893 & 38 \\
$A_{12}$ & 0.734 & 8 \\
$A_{31}$ & 0.708 & 27 \\
\hline
\end{tabular}

The results of CIR safety evaluation were divided into 7 levels, namely, strongly safe, safe, slightly safe, neutral, slightly risky, risky, and strongly risky. According to the established CIR safety EIS, the results of CIR safety evaluation were taken as the goal layer, the three primary indices were taken as the criteria layer, and the secondary and tertiary indices under each primary index was treated as an alternative. The weight of each index was determined through analytic hierarchy process (APH). The weights of the three primary indices are denoted as $\Phi_{1}, \Phi_{2}$, and $\Phi_{3}$. The weights of the secondary and tertiary indices under each primary index are denoted similarly. For example, $\Phi 1=\{\varphi 11, \varphi 12, \varphi 13, \varphi 14\}$, and $\Phi 11=\{\varphi 111, \varphi 112, \varphi 113, \varphi 114\}$.

\subsection{FCE}

During the FCE, each primary index of the EIS is evaluated, with every secondary index under it as a single factor; each secondary index is evaluated with every tertiary index under it as a single factor. Taking primary index $A_{1}$ for instance, the set of memberships of its secondary indices can be established as Table 2 .

Table 2. The set of memberships of secondary indices under $A_{1}$

\begin{tabular}{cccccccc}
\hline & Strongly safe & Safe & Slightly safe & Neutral & Slightly risky & Risky & Strongly risky \\
\hline$A_{11}$ & $\mu_{11}$ & $\mu_{12}$ & $\mu_{13}$ & $\mu_{14}$ & $\mu_{15}$ & $\mu_{16}$ & $\mu_{17}$ \\
$A_{12}$ & $\mu_{21}$ & $\mu_{22}$ & $\mu_{23}$ & $\mu_{24}$ & $\mu_{25}$ & $\mu_{26}$ & $\mu_{27}$ \\
$A_{13}$ & $\mu_{31}$ & $\mu_{32}$ & $\mu_{33}$ & $\mu_{34}$ & $\mu_{35}$ & $\mu_{36}$ & $\mu_{37}$ \\
$A_{14}$ & $\mu_{41}$ & $\mu_{42}$ & $\mu_{43}$ & $\mu_{44}$ & $\mu_{45}$ & $\mu_{46}$ & $\mu_{47}$ \\
\hline
\end{tabular}


Through AHP, the weights of secondary index $A_{11}$ can be obtained as $\Phi 11=\{\varphi 111, \varphi 112, \varphi 113, \varphi 114\}$. Let $M_{11}$ be single-factor evaluation matrix of $A_{11}$. Then, the evaluation results of $A_{11}$ can be expressed as:

$$
\begin{aligned}
& S_{S-11}=\Phi_{11} \cdot M_{11}=\left[\varphi_{11}, \varphi_{12}, \varphi_{13}, \varphi_{14}\right] \cdot\left[\begin{array}{ccc}
\mu_{11} & \cdots & \mu_{16} \\
\vdots & \ddots & \vdots \\
\mu_{41} & \cdots & \mu_{46}
\end{array}\right] \\
& =\left[S_{11}, S_{12}, S_{13}, S_{14}, S_{15}, S_{16}\right]
\end{aligned}
$$

where, $\varphi 111+\varphi 112+\varphi 113+\varphi 114=1$. Similarly, the evaluation results $S_{f-12}, S_{f-13}$, and $S_{f-14}$ of other secondary indices $A_{12}, A_{13}$ and $A_{14}$ under primary index $A_{1}$ can be expressed as:

$$
S_{1}=\left[\begin{array}{c}
S_{S-11} \\
\vdots \\
S_{S-41}
\end{array}\right]=\left[\begin{array}{ccc}
S_{11} & \cdots & S_{16} \\
\vdots & \ddots & \vdots \\
S_{41} & \cdots & S_{46}
\end{array}\right]
$$

As mentioned before, the weights of the secondary indices under primary index $A_{1}$ obtained by the AHP can be expressed as $\Phi 1=\{\varphi 1, \varphi 2, \varphi 3, \varphi 4\}$. Then, the FCE results of $A_{1}$ can be described as:

$$
\begin{aligned}
& F_{1}=\Phi_{1} \cdot S_{1}=\left[\varphi_{1}, \varphi_{2}, \varphi_{3}, \varphi_{4}\right] \cdot\left[\begin{array}{lll}
S_{11} & \cdots & S_{16} \\
\vdots & \ddots & \vdots \\
S_{41} & \cdots & S_{46}
\end{array}\right] \\
& =\left[E_{11}, E_{12}, E_{13}, E_{14}, E_{15}, E_{16}\right]
\end{aligned}
$$

The weights of the three primary indices on the criteria layer obtained by the AHP can be expressed as $\Phi=\{\Phi 1, \Phi 2, \Phi 3\}$. Similarly, the FCE results of the goal layer can be depicted as:

$$
\begin{aligned}
& \text { Security }=\Phi \cdot F_{1}=\left[\Phi_{1}, \Phi_{2}, \Phi_{3}\right] \cdot\left[\begin{array}{ccc}
F_{11} & \cdots & F_{16} \\
\vdots & \ddots & \vdots \\
F_{31} & \cdots & F_{36}
\end{array}\right] \\
& =\left[\operatorname{Sec}_{11}, \operatorname{Sec}_{12}, \operatorname{Sec}_{13}, \operatorname{Sec}_{14}, \operatorname{Sec}_{15}, \operatorname{Sec}_{16}\right]
\end{aligned}
$$

The FCE results of the goal layer were normalized. Then, the CIR safety was derived under the principle of maximum membership.

\section{ESTABLISHMENT OF RISK PROPAGATION MODEL}

The established CIR risk propagation model is shown in Figure 1. Based on the CIR indices, the process chain and value chain of information disclosure were reconstructed, and the links on the two chains were abstracted into risk propagation nodes. The paths between different links on the chains were abstracted as the edges between the nodes, forming a complex risk propagation network.

In the risk propagation network, every node is a risk carrier, and each edge is a risk propagation path. The carriers refer to all kinds of things that carry the risk flow of CIR, including tangible and intangible carriers. The tangible carriers include fund, material, and manpower of the company, while intangible carriers include policy, strategy, technology, and information.

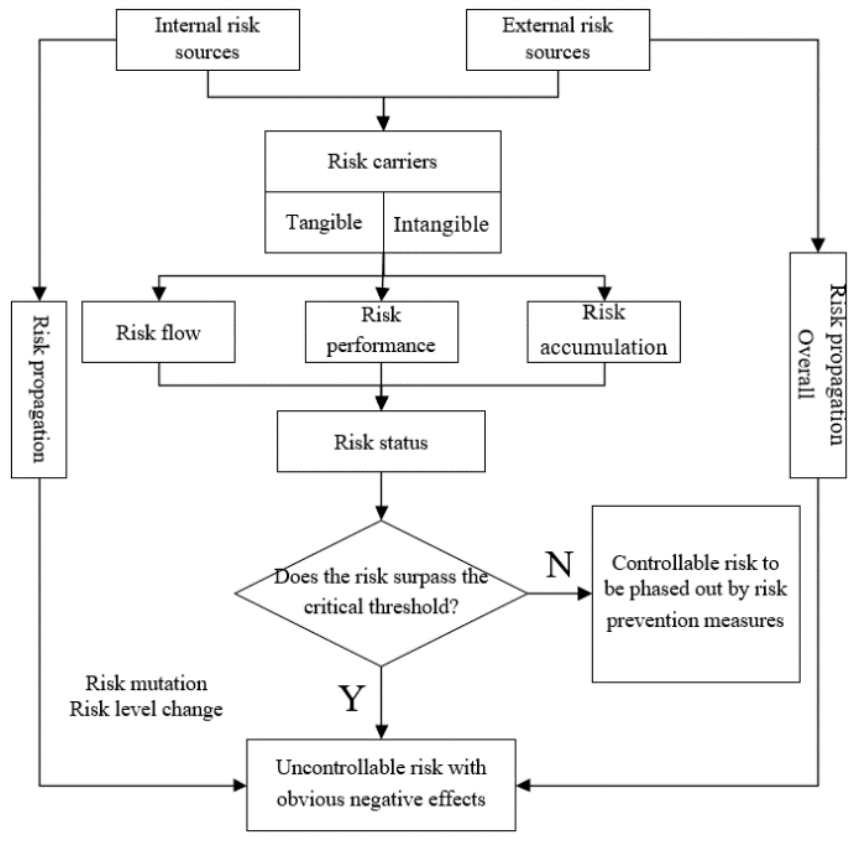

Figure 1. The CIR risk propagation model

The sources of CIR risks, either internal or external, exist on the overall propagation chain of the risk propagation network. It is from these risk sources that various CIR risks take shape, emerge, and start to propagate. The typical risk sources are internal opportunistic disclosure, safety of information propagation, changes in information propagation environment, and influence of mass media.

Based on the evaluation results of CIR safety, the risk adjustment threshold, risk release threshold, and risk control threshold could be determined, making risk measurement more dynamic.

\subsection{Hypotheses}

The propagation paths of CIR risk are formed based on the company's process chain and value chain of the information disclosure. The propagation process bears high resemblance with the three-stage transmission of infectious disease, which consists of an incubation period, an outbreak period, and a recovery period. The CIR risk propagation can be broken down into an internal propagation and accumulation period (internal period), an external amplification and enhancement period (external period), and a stabilization and recovery period (final period). All the nodes in the risk propagation network were divided as per the period of CIR risk propagation: the number of nodes in the internal, external, and final periods at time $t$ are denoted as $T A(t), A E(t)$, and $S R(t)$, respectively.

(1) It is assumed that the total number of nodes remains constant as $C=T A(t)+A E(t)+S R(t)$. The CIR risk carriers could be divided into system carriers, suppliers and customer carriers, financial personnel carriers, fund carriers, and financial information carriers. The number of these types of carriers are denoted as $C_{s}, C_{c}, C_{f}, C_{b}$, and $C_{i}$, in turn. Considering that the risks propagate along the paths between different links on the process chain and value chain, the degree of correlation between carriers was expressed by the connection weight $\omega_{a b}$.

(2) If two nodes have a connection weight of zero, the CIR risks will not flow between them. Let $\alpha_{a b}(t), \beta_{a b}(t)$, and $\gamma_{a b}(t)$ be the risk adjustment threshold, risk release threshold, and 
risk control threshold between nodes a and b, respectively. The three thresholds can be initialized as $\alpha_{0}(t)=\left[T A_{x 0}(t)+T A_{y 0}(t)+T A_{\mathrm{z} 0}(t)\right] / C$, $\beta_{0}(t)=\left[A E_{x 0}(t)+A E_{y 0}(t)+A E_{z 0}(t)\right] / C$, and $\gamma_{0}(t)=\left[S R_{x 0}(t)+S R_{y 0}(t)+S R_{\mathrm{z} 0}(t)\right] / C$, respectively. Suppose $\alpha_{a b}(t)$ is positively correlated with the correlation gradient $\rho_{a}(t)$ between the node and the process and value chains. Then, $\rho_{a}(t)=\left[T A_{\mathrm{a}}(t)+A E_{\mathrm{a}}(t)+S R_{\mathrm{a}}(t)\right] /\left[T A_{\mathrm{c}}(t)+A E_{c}(t)+S R_{c}(t)\right]$, where $T A_{\mathrm{c}}(t), A E_{c}(t)$, and $S R_{c}(t)$ are the risk adjustment threshold, risk release threshold, and risk control threshold of the company, respectively. The relationship between $\alpha_{a b}(t)$ and $\rho_{a}(t)$ can be described as $\alpha_{a b}(t)=\omega_{a b} \rho_{a}(t)$. During the internal period, node $a$ is affected by every node in the external period connected to it. This risk propagation effect can be expressed as $\alpha_{a}(t)=1$ $\prod\left(1-\alpha_{a b}(t)\right)$.

(3) At time $t$, the risk propagation probability $p_{1}(a, t)$ on the edge between node $a$ in the internal period and every node in the external period connected to it depends on $\alpha_{0}(t), \alpha_{a}(t)$, and the increment of risk propagation effect $\Delta \alpha_{a+}(t): p_{1}(a, t)=[1-$ $\left.\alpha_{0}(t)\right]\left[1-\Delta \alpha_{a+}(t)\right]\left[1-\prod\left(1-\alpha_{a b}(t)\right)\right]$. At time $t$, the state recovery probability $p_{2}(b, t)$ of node $b$ in the external period depends on $\beta_{0}(t)$, and the increment of state recovery ability $\Delta \beta_{b+}(t): p_{2}(b$, $t)=1-\left[1-\beta_{0}(t)\right]\left[1-\Delta \beta_{b+}(t)\right]$.

\subsection{Risk propagation model}

According to the above hypotheses, the risk propagation paths are affected by the reconstruction strategy of the process chain and value chain. At time $t$, node $a$ in the internal period might enter the risk state at the risk propagation probability of $p_{1}(a, t)$. The loss $\Delta \alpha_{a-}(t)$ of influence $\alpha_{a}(t)$ of node $a$ is positively correlated with the risk propagation probability. At time $t$, node $b$ in the external period might change from the risk state to the normal state at the state recovery probability of $p_{2}(b$, $t)$. The loss $\Delta \beta_{b-}(t)$ of state recovery ability $\beta_{b}(t)$ of node $b$ is negatively correlated with the state recovery probability.

According to the above analysis, the CIR risk propagation could be effectively managed, sub-effectively managed, or out of control. Based on the previous hypotheses, the CIR risk propagation can be modeled as:

$$
\left\{\begin{array}{l}
\frac{d T A(t)}{d t}=-p_{1}(a, t) T A(t) A E(t) \\
\frac{d A E(t)}{d t}=p_{1}(a, t) T A(t) A E(t)-p_{2}(a, t) A E(t) \\
\frac{d S R(t)}{d t}=p_{2}(a, t) A E(t)
\end{array}\right.
$$

Formula (6) must satisfy the following constraints:

$$
\left\{\begin{array}{c}
0 \leq \int \alpha_{a}(t) d t-\Delta \alpha_{a}(i) \leq 1 \\
0 \leq p_{1}(a, t) \leq 1 \\
0 \leq p_{2}(a, t) \leq 1
\end{array}\right.
$$

\subsection{Model derivation}

As shown in formula (5), the above set of differential equations are linked by the changes in the number of nodes $T A(t), A E(t)$, and $S R(t)$ in the three periods of risk propagation. Then, the risk propagation probability $p_{1}(a, t)$ and state recovery probability $p_{2}(a, t)$ can be respectively solved by:

$$
\left\{\begin{array}{l}
p_{1}(a, t)=\left[1+\alpha_{0}(t)\right] \Pi\left(1-\alpha_{a b}(t)\right)+\left[1-\alpha_{0}(t)\right] \Delta \alpha_{a+}(t) \\
p_{2}(a, t)=\left[1-\beta_{0}(t)\right] \Delta \beta_{a+}(t)+\beta_{0}(t)
\end{array}\right.
$$

Based on the relationship $T A(t)=T A_{x}(t)+T A_{y}(t)+T A_{z}(t)$, the established CIR risk propagation model was decomposed in the risk propagation cycle $T$ and subject to integration. Suppose $\alpha_{a}(t)$ reaches the ideal state in cycle $T$ through the reconstruction of process and value chains. Then, $\int \prod(1-$ $\left.\alpha_{a b}(t)\right) \mathrm{d} t=0, \int \Delta \alpha_{a+}(t) \mathrm{d} t \rightarrow 0$, and $\int \Delta \beta_{b^{+}}(t) \mathrm{d} t \rightarrow 0$. On this basis, the evolution of $T A$ in the internal period can be simplified as:

$$
\begin{aligned}
& \frac{\left(1+\omega_{a} \rho_{a}\right) \alpha_{0}(t)}{\omega_{a} \rho_{a}} T A_{x}(t)+\frac{1+\alpha_{0}(t)}{\omega_{a} \rho_{a}} T A_{y}(t) \\
& +\left(1-\alpha_{0}(t)\right) T A_{z}(t)+2 \beta_{0}(t)-1=0
\end{aligned}
$$

Based on the relationship $T A(t)=T A_{x}(t)+T A_{y}(t)+T A_{z}(t)$, the established CIR risk propagation model was decomposed in the risk propagation cycle $T$ and subject to integration, revealing the evolution of $A E(t)$ in the external period:

$$
\begin{aligned}
& \frac{1-\alpha_{0}(t)}{\omega_{a} \rho_{a}} A E_{x}(t)+\frac{\left(1+\omega_{a} \rho_{a}\right) \alpha_{0}(t)}{\omega_{a} \rho_{a}} A E_{y}(t) \\
& +\left(\alpha_{0}(t)-\beta_{0}(t)\right) A E_{z}(t)-\frac{\beta_{0}(t)}{2}=0
\end{aligned}
$$

Based on the relationship $S R(t)=S R_{x}(t)+S R_{y}(t)+S R_{z}(t)$, the established CIR risk propagation model was decomposed in the risk propagation cycle $T$ and subject to integration, revealing the evolution of $S R(t)$ in the final period:

$$
\begin{aligned}
& \frac{1-\alpha_{0}(t)}{\omega_{a} \rho_{a}} S R_{x}(t)+\frac{1-\alpha_{0}(t)}{\omega_{a} \rho_{a}} S R_{y}(t) \\
& +\frac{\left(1+\omega_{a} \rho_{a}\right) \beta_{0}(t)}{\omega_{a} \rho_{a}} S R_{z}(t)-\beta_{0}(t)=0
\end{aligned}
$$

By deriving $p_{1}(a, t)$ and $p_{2}(a, t)$ separately, the mean risk propagation rate and mean state recovery rate of cycle $T$ can be respectively obtained by:

$$
\left\{\begin{array}{l}
\frac{d p_{1}(a, t)}{d t}=-\left[1-\alpha_{0}(t)\right] \frac{d\left(1-\alpha_{a}(t)\right)}{d t} \\
-\left[1+\alpha_{0}(t)\right] \frac{d \Delta \alpha_{a+}(a, t) \alpha_{i}(t)}{d t} \\
\frac{d p_{2}(a, t)}{d t}=\left[1-\beta_{0}(t)\right] \frac{d \Delta \beta_{a+}(a, t)}{d t}
\end{array}\right.
$$

\section{BPNN-BASED PREDICTION OF RISK PROPAGATION PATHS}

\subsection{BPNN construction}

To make reasonable prediction of CIR risk propagation paths, this paper creates a multilayer feedforward NN with error backpropagation called the BPNN. The BPNN generally contain one input layer, $\mathrm{n}$ hidden layers, and one output layer. Assuming that only one predicted path is outputted, then the BPNN can be simplified as the multi-input, single-output network with only one hidden layer in Figure 2. Taking the 
$T A(t), A E(t)$, and $S R(t)$ values of the risk propagation model in Section 3 and the real-time changes of $\alpha_{a b}(t), \beta_{a b}(t), \gamma_{a b}(t), p_{1}(a$, $t)$, and $p_{2}(a, t)$ of each node as the inputs of the BPNN, an input vector can be constructed as:

$$
x_{i}=\left(x_{i-1}, x_{i-2}, \ldots, x_{i-N}\right)^{T}
$$

where, $i$ is the serial number of the reconstruction processes of process and value chains; $N$ is the number of input layer nodes. The hidden layer input can be expressed as:

$$
h_{I-j}=\sum_{i=1}^{N} \varepsilon_{i j} x_{i}-\lambda_{j}
$$

where, $\varepsilon_{i j}$ is the connection weight between the $\mathrm{i}$-th input layer node and the $\mathrm{j}$-th hidden layer node; $\lambda_{j}$ is the threshold of the $\mathrm{j}$-th hidden layer node. Then, the hidden layer output can be expressed as:

$$
h_{O-j}=\frac{1}{1+e^{-h_{j}}}
$$

The input of the k-th output layer node can be expressed as:

$$
g_{k}=\sum_{j=1}^{M} \eta_{j k} h_{O-j}-\delta_{k}
$$

where, $\eta_{j k}$ is the connection weight between the $\mathrm{j}$-th hidden layer node and the $\mathrm{k}$-th output layer node; $\delta_{k}$ is the threshold of the k-th output layer node. Thus, the output of the output layer can be calculated by:

$$
y_{k}=\frac{1}{1+e^{-g_{k}}}
$$

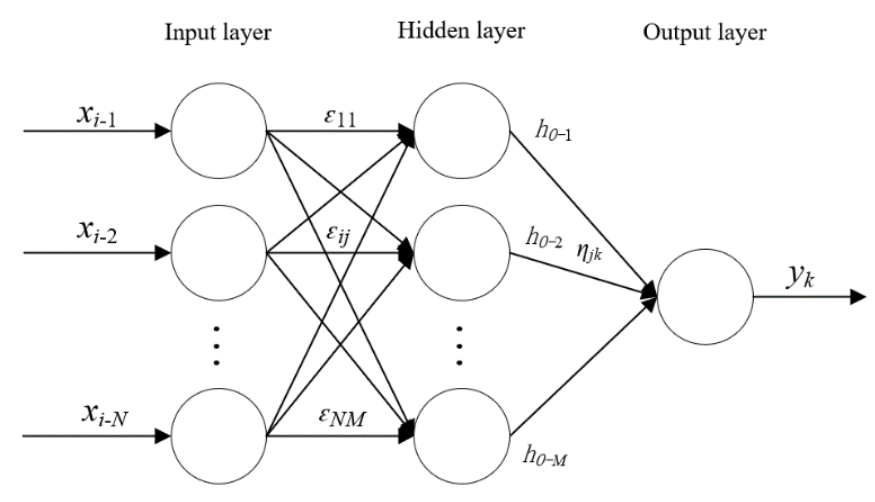

Figure 2. The structure of the BPNN

\subsection{BPNN training algorithm}

The BPNN designed to predict the CIR risk propagation path was trained in six steps:

Step 1. Randomly initialize the connecting weights $\varepsilon_{i j}$ and $\eta_{j k}$, and thresholds $\lambda_{j}$ and $\delta_{k}$ in the interval of $[-1,1]$, and import the sample data into the network.
Step 2. Calculate the input $h_{I-j}$ and output $h_{O-j}$ of the hidden layer, as well as the input $g_{k}$ and output $y_{k}$ of the output layer, according to formulas (13) and (14).

Step 3. Describe the mean squared error (MSE) between the expected output $\hat{y}_{k}$ and the actual output $y_{k}$ as:

$$
V_{t}=\frac{1}{2} \sum_{k=1}^{M} \varphi_{k}^{2}=\frac{1}{2} \sum_{k=1}^{M}\left(\hat{y}_{k}-y_{k}\right)^{2}
$$

Calculate the negative value of the partial derivative of $V_{t}$ to $g_{k}$ by:

$$
R_{t-k}=-\frac{\partial V_{t}}{\partial g_{k}}=\varphi_{k} y_{k}\left(1-y_{k}\right)=\left(\hat{y}_{k}-y_{k}\right) y_{k}\left(1-y_{k}\right)
$$

Calculate the negative value of the partial derivative of $V_{t}$ to hidden layer input $g_{k}$ by:

$$
E_{t-j}=-\frac{\partial V_{t}}{\partial h_{I-j}}=\left(\sum_{t=1}^{M} R_{t-k} \eta_{j k}\right) h_{O-j}\left(1-h_{O-j}\right)
$$

Step 4. Let $\mathrm{n}$ be the number of iterations, and $\tau_{1}$ and $\tau_{2}$ be the correction coefficients. Then, update $\eta_{j k}$ and $\delta_{k}$ respectively by:

$$
\begin{gathered}
\eta_{j k}(n+1)=\eta_{j k}(n)+\tau R_{t-k} h_{O-j} \\
\delta_{k}(n+1)=\delta_{k}(n)-\tau R_{t-k}
\end{gathered}
$$

Update $\varepsilon_{i j}$ and $\lambda_{j}$ respectively by:

$$
\begin{gathered}
\varepsilon_{i j}(n+1)=\varepsilon_{i j}(n)+\tau_{2} E_{t-j} x_{i} \\
\lambda_{j}(n+1)=\lambda_{j}(n)-\tau_{2} E_{t-j}
\end{gathered}
$$

Step 5. Randomly select the parameters of the risk propagation model for the next reconstruction cycle, and import them to the network. Return to Step 4 until all samples are trained.

Step 6. Perform a new round of training until $V_{t}$ falls in the interval of $[-\gamma, \gamma]$, where $\gamma$ is the preset training accuracy.

\section{EXPERIMENTS AND RESULTS ANALYSIS}

Based on the data disclosed by a company in its CIR 2019, this section carries out experiments on the reconstruction of process and value chains. During the reconstruction, the cycles, number of risk carriers, and types of risk carriers were collected, grouped into sample data with month as the time unit, and used to build a risk propagation network.

Table 3 provides the Cronbach's alphas of the tertiary indices under each criterion after reliability analysis on SPSS 21.0. It can be seen that the Cronbach's alphas of the tertiary indices averaged at 0.7 , indicating the tertiary indices have good overall reliability. 
Table 3. The Cronbach's alphas of the tertiary indices under each criterion

\begin{tabular}{|c|c|c|c|c|c|c|c|c|c|}
\hline Index & $\begin{array}{c}\text { Cronbach's } \\
\text { alpha } \\
\end{array}$ & Index & $\begin{array}{c}\text { Cronbach's } \\
\text { alpha } \\
\end{array}$ & Index & $\begin{array}{c}\text { Cronbach's } \\
\text { alpha }\end{array}$ & Index & $\begin{array}{c}\text { Cronbach's } \\
\text { alpha } \\
\end{array}$ & Index & $\begin{array}{c}\text { Cronbach's } \\
\text { alpha }\end{array}$ \\
\hline$A_{111}$ & 0.830 & $A_{121}$ & 0.656 & $A_{141}$ & 0.756 & $A_{221}$ & 0.547 & $A_{311}$ & 0.691 \\
\hline$A_{111}$ & 0.567 & $A_{131}$ & 0.669 & $A_{141}$ & 0.746 & $A_{221}$ & 0.856 & $A_{311}$ & 0.901 \\
\hline$A_{111}$ & 0.574 & $A_{131}$ & 0.745 & $A_{141}$ & 0.742 & $A_{221}$ & 0.641 & $A_{311}$ & 0.785 \\
\hline$A_{111}$ & 0.412 & $A_{131}$ & 0.751 & $A_{141}$ & 0.845 & $A_{221}$ & 0.875 & & \\
\hline$A_{121}$ & 0.689 & $A_{131}$ & 0.655 & $A_{211}$ & 0.941 & $A_{221}$ & 0.745 & & \\
\hline$A_{121}$ & 0.745 & $A_{131}$ & 0.698 & $A_{211}$ & 0.679 & $A_{221}$ & 0.756 & & \\
\hline$A_{121}$ & 0.874 & $A_{131}$ & 0.877 & $A_{211}$ & 0.697 & $A_{311}$ & 0.814 & & \\
\hline$A_{121}$ & 0.745 & $A_{141}$ & 4.33 & $A_{211}$ & 0.845 & $A_{311}$ & 0.615 & & \\
\hline
\end{tabular}

Table 4. The parameters of CIR risk propagation model

\begin{tabular}{ccccccccc}
\hline $\begin{array}{c}\text { No. of } \\
\text { reconstruction } \\
\text { process }\end{array}$ & $\begin{array}{c}\text { Scale of CIR } \\
\text { core subjects }\end{array}$ & $\begin{array}{c}\text { Degree of correlation } \\
\text { between risk carriers }\end{array}$ & $\boldsymbol{C}$ & $\boldsymbol{\omega}_{\boldsymbol{a} \boldsymbol{b}}$ & $\boldsymbol{\alpha}_{\mathbf{0}}$ & $\boldsymbol{\beta}_{\mathbf{0}}$ & $\begin{array}{c}\text { Mean risk } \\
\text { propagation } \\
\text { probability }\end{array}$ & $\begin{array}{c}\text { Mean state } \\
\text { recovery } \\
\text { probability }\end{array}$ \\
\hline 1 & 0.16 & 0.54 & 29 & 5 & 0.19 & 0.26 & 0.84 & 0.43 \\
2 & 0.21 & 0.57 & 33 & 14 & 0.29 & 0.22 & 0.71 & 0.45 \\
3 & 0.22 & 0.62 & 42 & 17 & 0.36 & 0.29 & 0.69 & 0.55 \\
4 & 0.35 & 0.66 & 59 & 26 & 0.39 & 0.24 & 0.51 & 0.58 \\
5 & 0.39 & 0.70 & 70 & 28 & 0.32 & 0.33 & 0.54 & 0.57 \\
6 & 0.51 & 0.76 & 83 & 31 & 0.31 & 0.29 & 0.45 & 0.47 \\
7 & 0.60 & 0.80 & 69 & 26 & 0.41 & 0.35 & 0.47 & 0.67 \\
8 & 0.61 & 0.83 & 88 & 39 & 0.39 & 0.39 & 0.46 & 0.73 \\
9 & 0.74 & 0.87 & 96 & 42 & 0.34 & 0.30 & 0.41 & 0.79 \\
10 & 0.85 & 0.89 & 115 & 47 & 0.30 & 0.35 & 0.37 & 0.34 \\
\hline
\end{tabular}

Next, the correlation gradient $\rho_{c}$ was set to 1 for the reconstruction of core subjects of the CIR; the risk adjustment threshold $\alpha_{c-0}(t)$, risk release threshold $\beta_{c-0}(t)$, and risk control threshold $\gamma_{c-0}(t)$ of the core subjects were all initialized as 0.5 ; the $\alpha_{0}(t), \beta_{0}(t)$, and $\gamma_{0}(t)$ of other nodes were proportionally reduced by the gradient $\rho$ of the reconstruction process. Table 4 lists the parameters of CIR risk propagation model after the reconstruction of process and value chains.

Based on the hypotheses and parameter settings of our model, the reconstruction experiments were carried out, assuming that CIR risk propagation can be effectively controlled, that is, under the most ideal state out of the three possible states of risk propagation.

With the aid of Matlab, M functions Evo1.m, Evo2.m and Evo3.m and the $\mathrm{M}$ executable file Evorun.m were compiled for the results (8), (9), and (10) of CIR risk propagation in each period. The $\mathrm{M}$ functions were executed $60-80$ times, and the results were combined and averaged. Figures 3, 4, and 5 display the results of CIR risk propagation in the internal, external, and final periods, respectively.

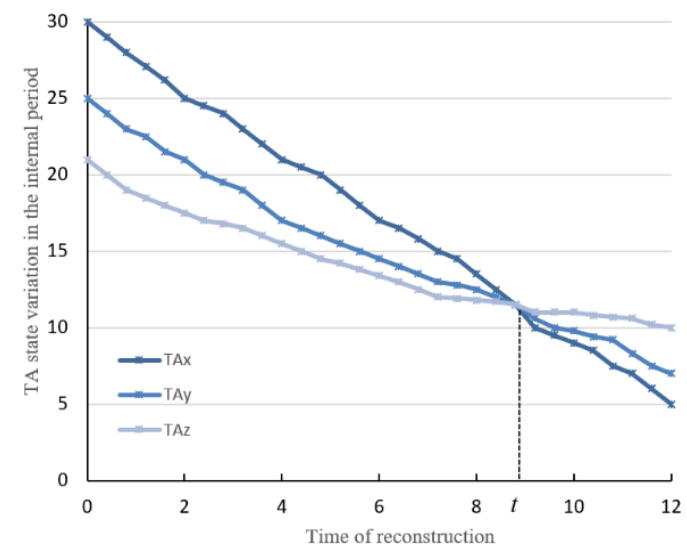

Figure 3. The $T A$ state variation in the internal period
As shown in Figure 3, during the internal period, the $T A(t)$ value decreased gently, and the CIR risk propagation reached the equilibrium at the intersection. Before the intersection, the reconstruction focuses on the prewarning of risk propagation; after the intersection, the reconstruction focuses on the restoration from the risk state. It can be seen that the keys of the reconstruction of the process and value chains lie in the reconstruction of the risk propagation prewarning module and risk state restoration module. During the reconstruction of risk propagation prewarning module, the risk flow direction and correlation between risk carriers directly bear on the risk control effect.

As shown in Figure 4, during the external period, the $A E$ state exhibited as a parabolic curve. The left intersection is the critical point, at which risk propagation occurred between two risk carriers; the right intersection is the critical point, at which the risk diffusion changed into risk convergence. The left intersection was slightly higher than the right intersection. This means the overall safety of the CIR increases rapidly through the reconstruction cycle.

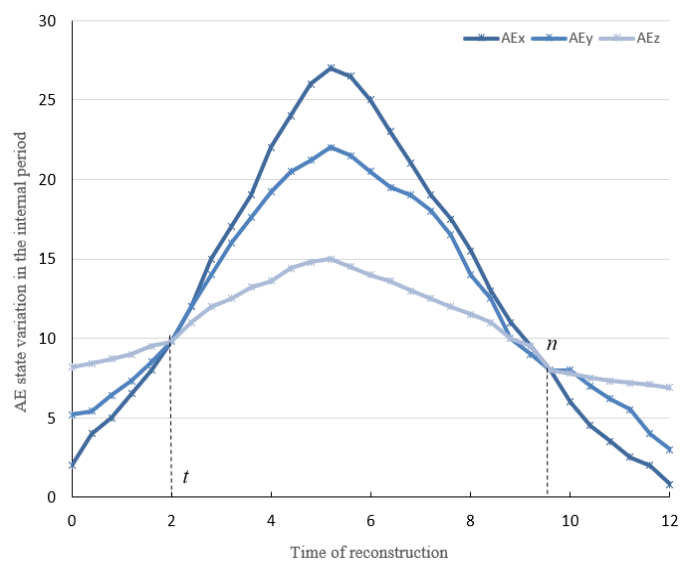

Figure 4. The $A E$ state variation in the internal period 


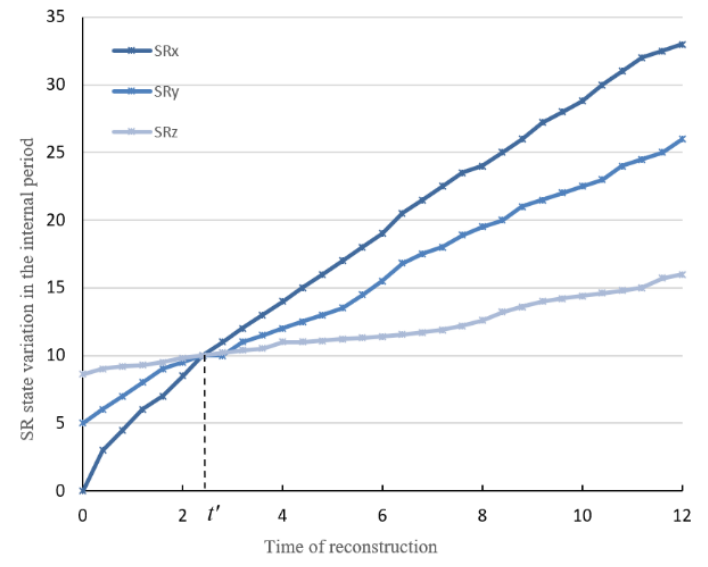

Figure 5. The $S R$ state variation in the internal period

As shown in Figure 5, during the final period, the intersection is the critical point, at which the risk propagation between two risk carriers gradually stabilized and returned to the normal state. Before the intersection, the CIR was highly risky; after the intersection, the CIR risks were under control, i.e., the CIR was slightly safe. At the beginning of the final period, many nodes needed to be restored in the risk propagation network, and the recovery rate was rather slow. As more and more nodes were restored, the reconstruction process was simplified, and the node recovery gradually picked up speed. This means the CIR risk propagation has relatively high fluidity.

In addition, Figures 3-5 confirm the following finding from Table 4: when the CIR risk propagation is effectively controlled, the mean risk propagation probability of all risk carriers is on the decline, while the mean state recovery probability is on the rise.

The next task is to verify the prediction effect of our BPNN on CIR risk propagation path. For this purpose, 200 sets of $T A(t), A E(t)$, and $S R(t)$ of CIR risk propagation model and the $\alpha_{a b}(t), \beta_{a b}(t), \gamma_{a b}(t), p_{1}(a, t)$, and $p_{2}(a, t)$ values of each node were imported to the BPNN. Only one node output, which falls in $[-1,1]$, was allowed on the output layer, representing the overall evaluation result on the reconstruction and control effect of CIR risk propagation path.

The BPNN was established on Matlab, including 48 input layer nodes, 24 hidden layer nodes, and 1 output layer node. As shown in Figures 6-8, the BPNN entered the convergence state and reach the preset training accuracy of $10^{-6}$, after 800 trainings. Besides, the BPNN predicted the CIR risk propagation path fairly accurately.

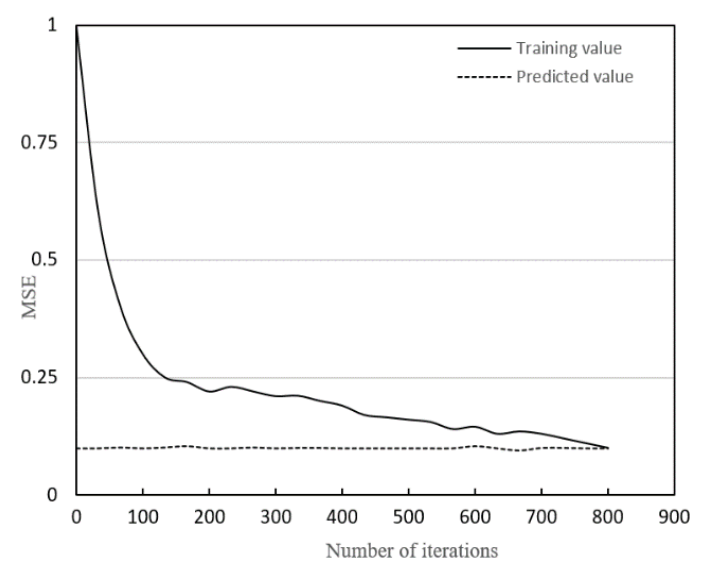

Figure 6. The MSE of prediction results

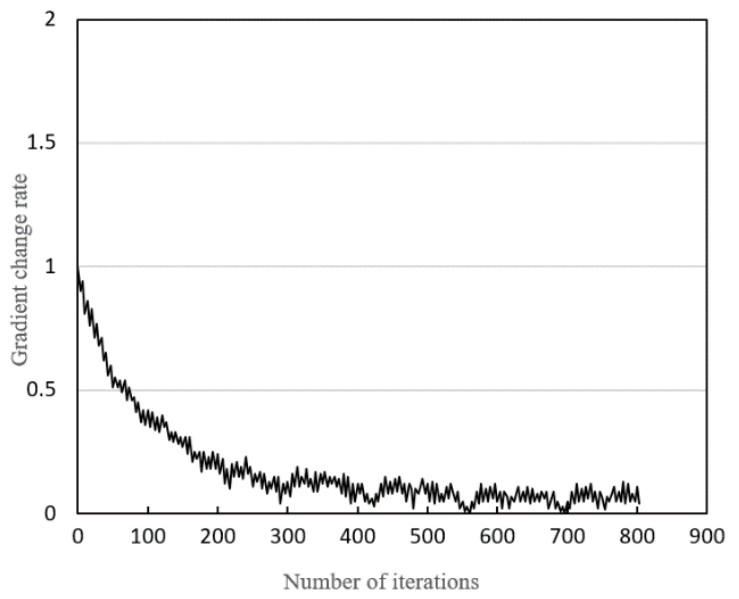

Figure 7. The simulated results on correlation gradient

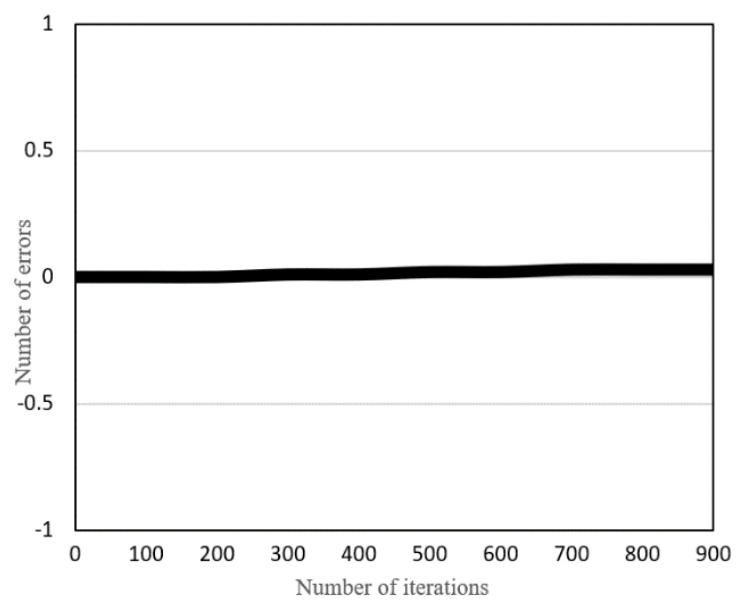

Figure 8. The simulation results on prediction errors

\section{CONCLUSIONS}

This paper mainly presents an CIR risk propagation model based on the FNN. Firstly, an CIR safety EIS was created, and each of its index was subject to reliability analysis and weight calculation. Then, an FCE was carried out on the entire EIS. Through experiments, the Cronbach's alphas of the secondary and tertiary indices under each criterion were obtained, after SPSS reliability analysis. Based on the evaluation results, the hypotheses and modes of risk propagation were summarized, creating the CIR risk propagation model. Several experiments were conducted on CIR risk propagation to disclose the variations of $T A(t), A E(t)$, and $S R(t)$ in different periods of the reconstruction process. The observed results confirm that the proposed model becomes increasingly safe through the reconstruction. Finally, a BPNN was constructed to predict the risk propagation path of the proposed model. Experimental results demonstrate that the BPNN can converge within the preset number of iterations, and make accurate predictions of CIR risk propagation path.

\section{ACKNOWLEDGMENT}

The Paper is supported by Fujian Social Science Fund Project: Research on Risk Ripple Effect and Crisis Management of Corporate Internet Reporting Based on SAR Framework (Grant No.: FJ2017C015). 


\section{REFERENCES}

[1] Stergiaki, E., Vazakidis, A., Stavropoulos, A. (2015). Development and evaluation of a prototype web XBRLenabled financial platform for the generation and presentation of financial statements according to IFRS. International Journal of Accounting and Taxation, 31: 74-101. https://doi.org/10.15640/ijat.v3n1a5

[2] Martić, V. (2015). Application of XBRL-A in the function of improvement of quality of financial reporting in montenegro. Facta Universitatis-Economics and Organization, 12(4): 279-295.

[3] Skorupski, J. (2016). The simulation-fuzzy method of assessing the risk of air traffic accidents using the fuzzy risk matrix. Safety Science, 88: 76-87. https://doi.org/10.1016/j.ssci.2016.04.025

[4] Blankespoor, E., Miller, B.P., White, H.D. (2014). Initial evidence on the market impact of the XBRL mandate. Review of Accounting Studies, 19(4): 1468-1503. https://doi.org/10.1007/s11142-013-9273-4

[5] Efendi, J., Park, J.D., Smith, L.M. (2014). Do XBRL filings enhance informational efficiency? Early evidence from post-earnings announcement drift. Journal of Business Research, 67(6): 1099-1105. https://doi.org/10.1016/j.jbusres.2013.05.051

[6] Dijcks, J.P. (2012). Oracle: Big data for the enterprise. Oracle white paper, 16.

[7] Vasarhelyi, M.A., Chan, D.Y., Krahel, J.P. (2012). Consequences of XBRL standardization on financial statement data. Journal of Information Systems, 26(1): 155-167. https://doi.org/10.2308/isys-10258

[8] Janssen, M., van Veenstra, A.F., Van Der Voort, H. (2013). Management and failure of large transformation projects: Factors affecting user adoption. In International Working Conference on Transfer and Diffusion of IT, pp. 121-135. https://doi.org/10.1007/978-3-642-38862-0 8

[9] Harris, T.S., Morsfield, S.G. (2012). An evaluation of the current state and future of XBRL and interactive data for investors and analysts. https://doi.org/10.7916/D8CJ8NV2

[10] Alles, M., Piechocki, M. (2012). Will XBRL improve corporate governance? A framework for enhancing governance decision making using interactive data. International Journal of Accounting Information Systems, 13(2): 91-108. https://doi.org/10.1016/j.accinf.2010.09.008

[11] Seele, P. (2016). Digitally unified reporting: how XBRLbased real-time transparency helps in combining integrated sustainability reporting and performance control. Journal of Cleaner Production, 136: 65-77. https://doi.org/10.1016/j.jclepro.2016.01.102

[12] Kaya, D., Pronobis, P. (2016). The benefits of structured data across the information supply chain: Initial evidence on XBRL adoption and loan contracting of private firms. Journal of Accounting and Public Policy, 35(4): 417-436. https://doi.org/10.1016/j.jaccpubpol.2016.04.003

[13] Santos, I., Castro, E., Velasco, M. (2016). XBRL formula specification in the multidimensional data model. Information $\quad$ Systems, 20-37. https://doi.org/10.1016/j.is.2015.11.001

[14] Lowe, A., Locke, J., Lymer, A. (2012). The SEC's retail investor 2.0: Interactive data and the rise of calculative accountability. Critical Perspectives on Accounting, 23(3):

183-200.

https://doi.org/10.1016/j.cpa.2011.12.004

[15] Chen, B. (2019). Financial Market Reform in China: Progress, Problems, and Prospects. Routledge, ISBN: 0813336198

[16] Tsantekidis, A., Passalis, N., Tefas, A., Kanniainen, J., Gabbouj, M., Iosifidis, A. (2017). Forecasting stock prices from the limit order book using convolutional neural networks. In 2017 IEEE 19th Conference on Business Informatics (CBI), pp. 7-12. https://doi.org/10.1109/CBI.2017.23

[17] Breuel, T.M. (2017). High performance text recognition using a hybrid convolutional-LSTM implementation. In 2017 14th IAPR International Conference on Document Analysis and Recognition (ICDAR), Kyoto, pp. 11-16. https://doi.org/10.1109/ICDAR.2017.12

[18] Schmidhuber, J. (2015). Deep learning in neural networks: An overview. Neural Networks, 61: 85-117. https://doi.org/10.1016/j.neunet.2014.09.003

[19] Ribas, J.R., Arce, M.E., Sohler, F.A., Suárez-García, A. (2019). Data and calculation approach of the fuzzy AHP risk assessment of a large hydroelectric project. Data in Brief, 25: 104294. https://doi.org/10.1016/j.dib.2019.104294

[20] Negnevitsky, M., Nguyen, D.H., Piekutowski, M. (2014) Risk assessment for power system operation planning with high wind power penetration. IEEE Transactions on Power Systems, 30(3): 1359-1368. https://doi.org/10.1109/TPWRS.2014.2339358

[21] Centonze, P., Kim, D.Y., Kim, S. (2019). Security and privacy frameworks for access control big data systems. Comput. Mater. Continua, 59(2): 361-374. https://doi.org/10.32604/cmc.2019.06223

[22] Dineshreddy, V., Gangadharan, G.R. (2016). Towards an "Internet of Things" framework for financial services sector. In 2016 3rd International Conference on Recent Advances in Information Technology (RAIT), pp. 177181. https://doi.org/10.1109/RAIT.2016.7507897

[23] Murjadi, M.D., Rustam, Z. (2018, December). Fuzzy support vector machines based on adaptive particle swarm optimization for credit risk analysis. In J. Phys., Conf. Ser., 1108(1): 1-6. https://doi.org/10.1088/17426596/1108/1/012052

[24] Cakra, Y.E., Trisedya, B.D. (2015). Stock price prediction using linear regression based on sentiment analysis. In 2015 International Conference on Advanced Computer Science and Information Systems (ICACSIS), pp.

147-154. https://doi.org/10.1109/ICACSIS.2015.7415179

[25] Shan, Y.G., Troshani, I., Richardson, G. (2015). An empirical comparison of the effect of XBRL on audit fees in the US and Japan. Journal of Contemporary Accounting \& Economics, 11(2): 89-103. https://doi.org/10.1016/j.jcae.2015.01.001 